\title{
Article \\ Epigenetic Age Acceleration Is Not Associated with Age-Related Macular Degeneration
}

\author{
Neil Saptarshi ${ }^{1,+}$, Daniel Green ${ }^{2,+}$, Angela Cree ${ }^{3}$, Andrew Lotery ${ }^{3}$, Luminita Paraoan ${ }^{1, * \mathbb{D}}$ \\ and Louise F. Porter 1,4,5,*iD
}

1 Department of Eye and Vision Science, Institute of Life Course and Medical Sciences, University of Liverpool, Liverpool L7 8TX, UK; N.saptarshi@liverpool.ac.uk

2 Department of Musculoskeletal Biology, Institute of Life Course and Medical Sciences, University of Liverpool, Liverpool L7 8TX, UK; daniel.green@liverpool.ac.uk

3 Faculty of Medicine, University of Southampton, Southampton SO16 6YD, UK; a.j.cree@soton.ac.uk (A.C.); A.J.Lotery@soton.ac.uk (A.L.)

4 Laboratoire de Génétique Médicale INSERM U1112, Institut de Génétique Médicale d'Alsace (IGMA), Université de Strasbourg, 67000 Strasbourg, France

5 Service de Génétique Médicale, Centre de Référence pour les Affections Rares en Génétique Ophtalmologique (CARGO), Institut de Génétique Médicale d'Alsace (IGMA), 67000 Strasbourg, France

* Correspondence: lparaoan@liverpool.ac.uk (L.P.); lfporter@unistra.fr (L.F.P.)

+ Authors contributed equally.

Citation: Saptarshi, N.; Green, D.; Cree, A.; Lotery, A.; Paraoan, L.; Porter, L.F. Epigenetic Age Acceleration Is Not Associated with Age-Related Macular Degeneration. Int. J. Mol. Sci. 2021, 22, 13457. https: / / doi.org/10.3390/ ijms222413457

Academic Editor: Janusz Blasiak

Received: 7 October 2021

Accepted: 2 December 2021

Published: 15 December 2021

Publisher's Note: MDPI stays neutral with regard to jurisdictional claims in published maps and institutional affiliations.

Copyright: (c) 2021 by the authors. Licensee MDPI, Basel, Switzerland. This article is an open access article distributed under the terms and conditions of the Creative Commons Attribution (CC BY) license (https:/ / creativecommons.org/licenses/by/ $4.0 /)$.

\begin{abstract}
DNA methylation age (DNAm age) estimation is a powerful biomarker of human ageing. To date, epigenetic clocks have not been evaluated in age-related macular degeneration (AMD). Here, we perform genome-wide DNA methylation analyses in blood of AMD patients with a documented smoking history (14 AMD, 16 Normal), identifying loci of differential methylation (DML) with a relaxed $p$-value criterion $\left(p \leq 10^{-4}\right.$ ). We conduct DNAm age analyses using the Horvath-multi tissue, Hannum and Skin \& Blood epigenetic clocks in both blood and retinal pigment epithelium (RPE). We perform Ingenuity Pathway Analysis Causal Network Analysis (IPA CNA) on the topmost significantly differentially methylated $\mathrm{CPG}$ probes in blood and RPE. Results show poor performance of epigenetic clocks in RPE. Epigenetic age acceleration (EAA) was not observed in AMD. However, we observe positive EAA in blood of smokers, and in smokers with AMD. DML analysis revealed hypomethylation at cg04953735 within RPTOR $\left(p=6.51 \times 10^{-5} ; \Delta \beta=-11.95 \%\right)$. IPA CNA in the RPE also identified RPTOR as the putative master regulator, predicted to be inhibited in AMD. In conclusion, this is the first study evaluating an association of epigenetic ageing in AMD. We posit a role for RPTOR as a common master regulator of methylation changes in the RPE in AMD.
\end{abstract}

Keywords: age-related macular degeneration; epigenetic clock; DNA methylation; ageing; retinal pigment epithelium; whole blood

\section{Introduction}

Ageing is a complex physiological process characterised by progressive loss of tissue functionality and an increased risk of death [1]. DNA methylation (DNAm) is the most widely studied epigenetic modification in ageing [2]. Epigenetic clocks, which estimate chronological age based on DNA methylation age (DNAm age) are promising biomarkers of human ageing, designed around well-established patterns of DNAm changes over an individual's lifespan [2-5]. Several epigenetic clocks have been developed [6]. With few exceptions, most individual CpG probes selected for the epigenetic clocks have poor to moderate DNAm age predictive performance [7]. However, the weighted combination of many CpG probes, usually 300-400, results in highly accurate single and pan tissue DNAm age predictors [7]. Horvath's multi-tissue [3], Hannum's [4], and the Skin \& Blood epigenetic clocks [8] were constructed using penalised multivariate elastic net regression models that select $\mathrm{CpG}$ probes for each respective clock by minimising the residual error of 
predicting age while removing redundant coefficients from the model. Importantly, they differ based on tissue types used in clock training data $[3,4,8]$. From a biological perspective, the epigenetic clocks are believed to reflect the function of the "epigenetic maintenance system" (EMS), a measure of cumulative work used to maintain epigenetic stability in the genome [3]. A positive or negative deviation of estimated DNAm age from chronological age is termed "epigenetic age acceleration" (EAA). Positive EAA is physiologically necessary early in life, when rapid cellular development demands that epigenetic stability is maintained [7]. Positive EAA is also reported in individuals with age-related neurodegenerative diseases including Alzheimer's [9] and Parkinson's disease [10], in addition to some cancers [11]. However, significant negative EAA has been identified in the cerebellum of extremely elderly humans ( $>100$ years old) and in certain cancers [12-15], acting as a predictor of poor prognostic outcomes in the latter [15]. Together these studies indicate that both positive and negative accelerations of epigenetic age represent cellular adaptive responses to genomic/epigenomic instability, contributors to age-related disease [7].

Age-related macular degeneration (AMD) is a degenerative disease affecting the retinal pigment epithelium (RPE) and retina, representing a foremost cause of blindness with global projection estimates of 288 million cases by 2040 [16-18]. The greatest non-modifiable risk factor for AMD is advanced age [16], whereas smoking is the most significant modifiable risk factor [19-22]. Studies investigating a role for DNAm in AMD to date have primarily focused on site and region specific DNAm changes and their association with altered transcriptional profiles of individual genes [23-27]. Smoking as well as ageing exert direct effects on DNAm, with established DNAm changes occurring at specific CpG probes in blood of smokers [28,29]. Positive EAA calculated using Horvath's multi tissue clock has also been observed in lung tissues of smokers [30]. Therefore, major AMD risk factors, such as ageing and smoking, influence epigenetic ageing and EMS responses. However, to date, analysis of epigenetic ageing using the epigenetic clocks has not been applied to primary sites of pathogenesis in AMD including RPE, or even blood samples of patients with AMD. In addition, a link between smoking status and epigenetic ageing in AMD has not been investigated.

Epigenetic age estimations and DNAm signatures are often tissue-type dependent $[12,31]$. In this study, we investigate a role for epigenetic ageing in AMD by performing DNAm age estimations using the Horvath multi-tissue [3], Hannum [4], and Skin \& Blood [8] epigenetic clocks on age-matched RPE and whole blood from patients with AMD and healthy controls. The Horvath's multi-tissue and Skin \& Blood epigenetic clock were chosen due to favourable cross-tissue performance [3,8], and Hannum's clock because it was constructed using solely whole blood-derived data from a cohort spanning a large age range (19-101 years) [4]. All three were chosen based on alleged robustness of chronological age prediction $[3,4,8]$. Firstly, we assess the reliability of different epigenetic clocks in the RPE and blood. Secondly, we analysed EAA in Normal and AMD affected RPE and whole blood. We also conducted a secondary analysis assessing an association of smoking status with EAA in AMD whole blood. Furthermore, we conducted Ingenuity Pathway Analysis Causal Network Analysis (IPA CNA) in age-matched Normal and AMD-affected RPE and whole blood. We posit a role for RPTOR, which encodes the Regulatory Associated Protein of mTOR Complex 1 (Raptor) as a common master regulator of methylation changes in the RPE in AMD, which also displays differential methylation in whole blood of AMD patients.

\section{Results}

\subsection{Epigenetic Clocks Display Poor Performance in the RPE}

We performed DNAm age estimation analysis on RPE and whole blood-derived genomic DNA (gDNA) using the Horvath multi-tissue, Hannum, and Skin \& Blood epigenetic clocks. RPE DNAm data was acquired from our previously described cohort $(n=44)$ using the Illumina Infinium HumanMethylation450K BeadChip array (450K-array) [27]. Whole blood-derived gDNA methylation data was acquired using the Illumina Infinium Methyla- 
tionEPIC BeadChip array (EPIC-array) from the Southampton Cohort $(n=30)$. DNAm age estimations for all clocks assessed can be found in Supplementary Materials: Additional File S1. In RPE, Horvath's multi-tissue (Figure 1A), Hannum's (Figure 1B), and the Skin $\&$ Blood epigenetic clocks (Figure 1C) all performed poorly. Weak positive correlations were observed between DNAm age and chronological age. In whole blood-derived gDNA, Horvath's multi-tissue (Figure 1D), Hannum's (Figure 1E), and the Skin \& Blood clocks (Figure 1F) demonstrated better correlation between DNAm age and chronological age compared to RPE. This suggests both improved predictive capability and better calibration in whole blood. The Skin \& Blood clock (Figure 1F) displayed the most accurate DNAm age estimation of all three clocks assessed.

A

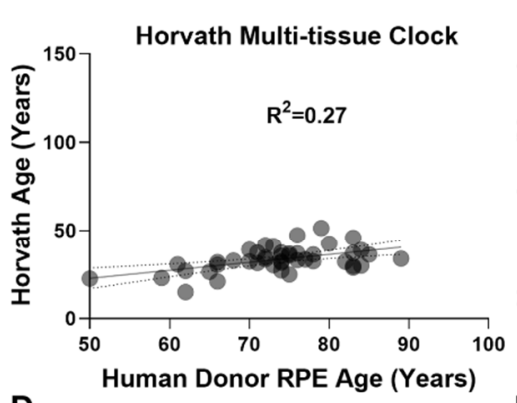

D

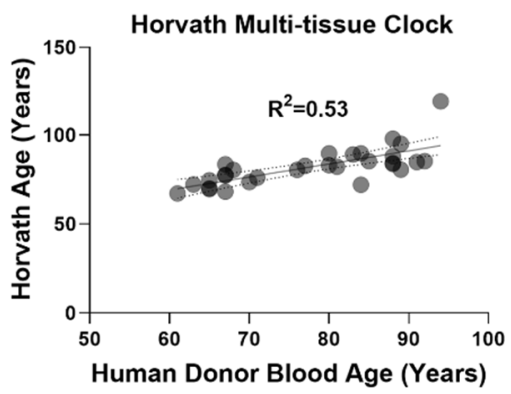

B

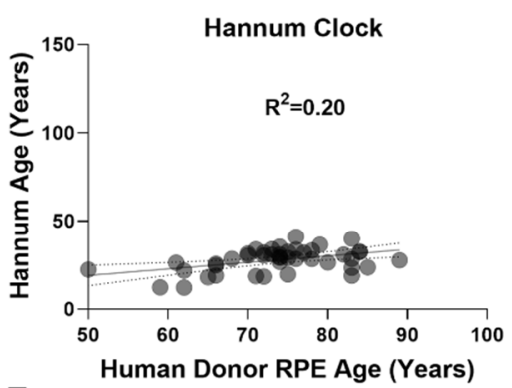

$\mathbf{E}$

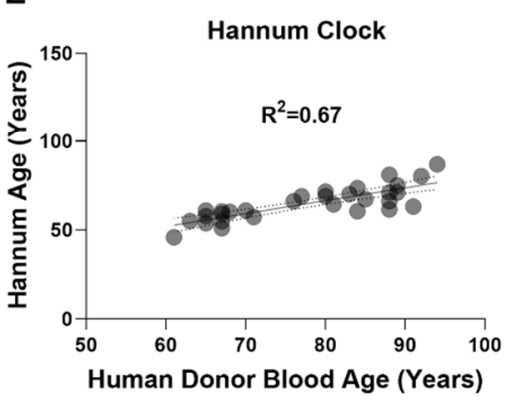

C
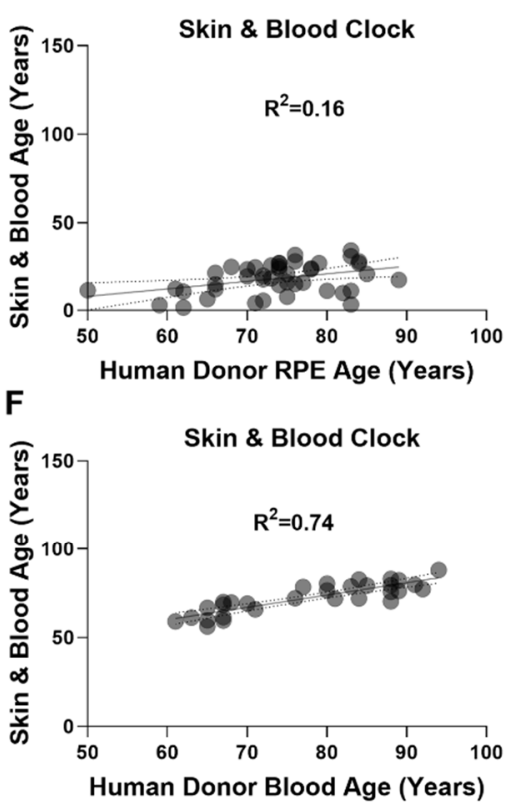

Figure 1. Epigenetic clocks perform poorly in RPE. Linear regressions of estimated DNAm age (years) with chronological age (years) were performed on $(\mathbf{A}-\mathbf{C})$ RPE $(n=44)$ and $(\mathbf{D}-\mathbf{F})$ whole blood-derived $(n=30)$ gDNA samples, to assess the performance of (A,D) Horvath's multi-tissue clock, (B,E) Hannum's clock, and (C,F) the Skin \& Blood clock. (A-C) In RPE gDNA, linear regressions of DNAm age estimation with chronological age are shown for: (A) Horvath's multi-tissue clock $\left(R^{2}=0.27\right)$; (B) Hannum's clock $\left(R^{2}=0.20\right)$; and $(C)$ Skin \& Blood clock $\left(R^{2}=0.16\right)$. All demonstrated poor clock performance and weak correlations. In whole blood-derived gDNA, linear regressions of DNAm age estimation with chronological age for (D) Horvath's multi tissue clock $\left(R^{2}=0.53\right)$, (E) Hannum's clock $\left(R^{2}=0.67\right)$, and the (F) Skin \& Blood clock $\left(R^{2}=0.74\right)$ demonstrated improved performance with the Skin \& Blood clock showing the most accurate predictions.

\subsection{Epigenetic Age Acceleration Is Not Associated with AMD}

We next investigated whether AMD patients displayed a positive or negative EAA when compared to Normal samples in both RPE [27] and whole blood-derived DNAm data. We define EAA as DNAm age minus chronological age. In human donor RPE, a marked and consistent negative mean EAA was observed in both Normal $(n=19)$ and AMD $(n=25)$ groups using Horvath's multi-tissue (Normal; -40.85 years; AMD: -39.73 years) (Figure 2A); Hannum's (Normal: -46.43 years; AMD: -45.38 years) (Figure 2B); and the Skin \& Blood clock (Normal: -56.50 years; AMD: -55.10 years) (Figure $2 \mathrm{C}$ ). When considering both Normal $(n=16)$ and AMD $(n=14)$ whole blood samples, Horvath's multitissue clock displayed a small increase in EAA (Normal: +5.07 years; AMD: +3.53 years) (Figure 2D); whereas the Hannum (Normal: -12.21 years; AMD: -13.69 years) (Figure 2E) and Skin \& Blood (Normal: -4.27 years; AMD: -6.78 years) clocks displayed decreases in EAA across groups (Figure 2F). No significant difference was observed in EAA between Normal and AMD groups across all clocks tested in the RPE (Figure 2A-C) and whole 
blood-derived gDNA (Figure 2D-F), suggesting no association of EAA with AMD across these tissues.

A
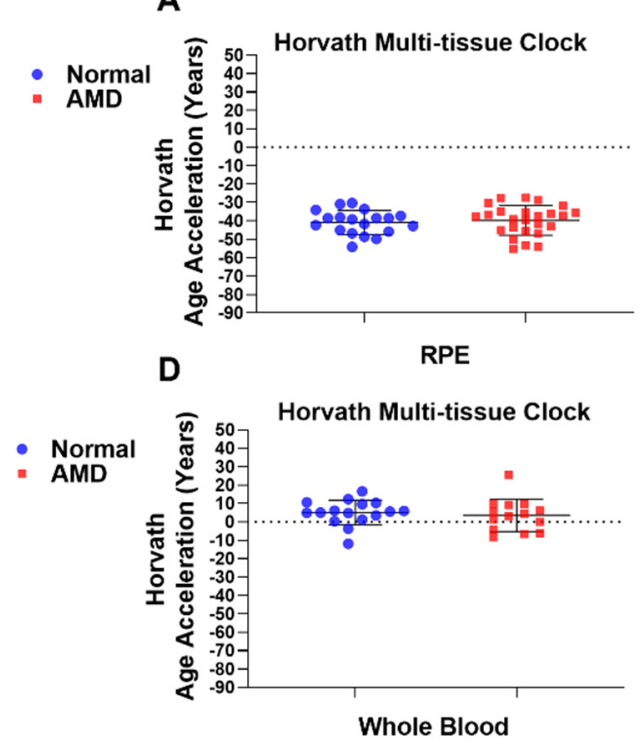

B

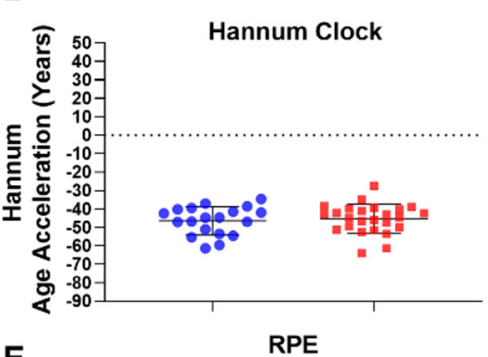

E

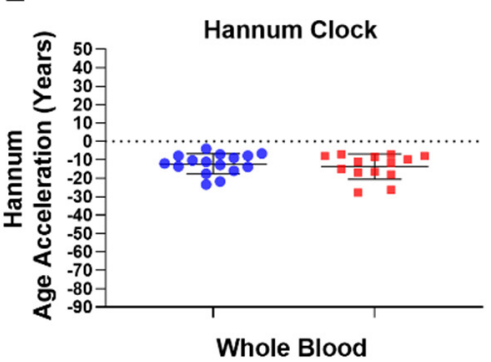

C
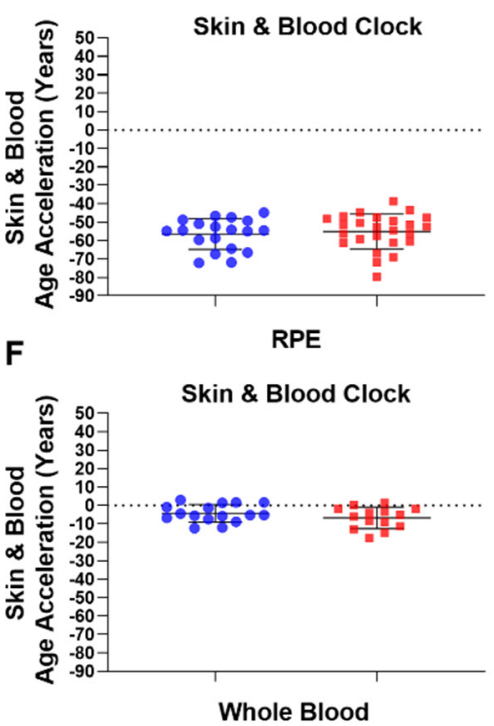

Figure 2. Epigenetic age acceleration is not associated with AMD. EAA analysis was conducted on AMD and Normal samples in (A-C) RPE (AMD: $n=25$; Normal: $n=19$ ) and (D-F) whole blood-derived gDNA (AMD: $n=14$; Normal: $n=16$ ) using (A,D) Horvath's multi-tissue clock; (B,E) Hannum's clock; and the (C,F) Skin \& Blood clock. No significant difference in EAA was found for RPE using (A) Horvath's multi-tissue clock ( $p=0.5109)$; (B) Hannum's clock $(p=0.7784)$; and the (C) Skin \& Blood clock ( $p=0.5733)$, or in whole blood-derived gDNA using (D) Horvath's multi-tissue clock $(p=0.3769)$; (E) Hannum's clock $(p=0.6374)$; or the $(\mathbf{F})$ Skin \& Blood clock $(p=0.2939)$. Statistical analysis was performed using a Mann-Whitney test.

\subsection{Smokers Display Increased Epigenetic Age Acceleration Compared to Non-Smokers}

Smoking is the most significant modifiable risk factor for AMD [19]. Information on smoking status was available for whole blood-derived gDNA samples from the Southampton Cohort. Therefore, we investigated the association of smoking with EAA in whole blood-derived gDNA using univariate analysis comparing Smokers and Non-Smokers. We found significant increases in EAA in Non-Smoker compared to Smoker groups using the Horvath multi-tissue epigenetic clock (Non-Smoker: -0.43 years; Smoker: +7.12 years) (Figure 3A). Using the Hannum (Non-Smoker: -17.26 years; Smoker: -10.38 years) (Figure 3B) and Skin \& Blood (Non-Smokers: -9.11 years; Smoker: -3.32 years) (Figure 3C) epigenetic clocks, we found larger mean negative EAAs across groups but still observed significant increases in EAA when comparing Non-Smoker and Smoker groups. 
A

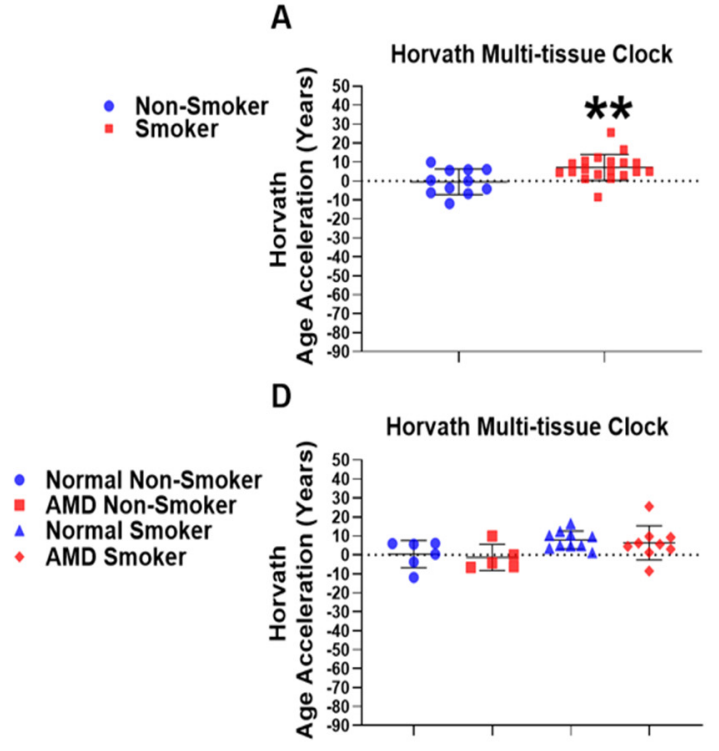

B

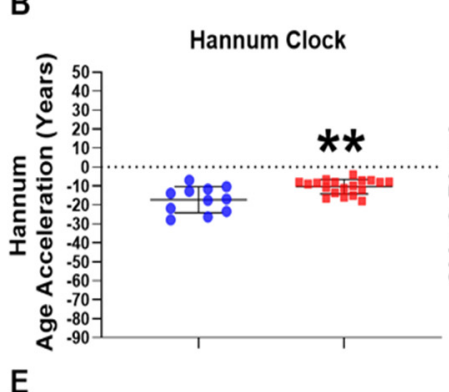

$\mathrm{E}$

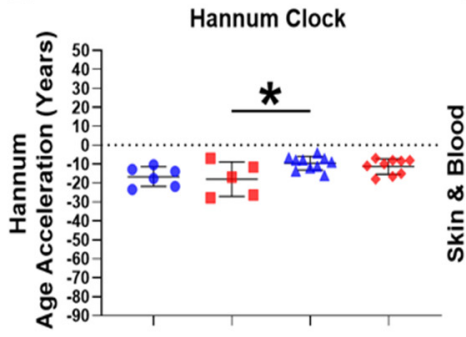

C
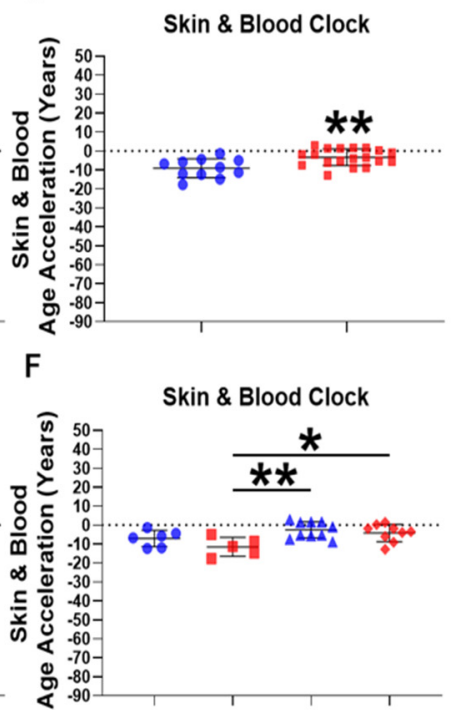

Figure 3. Epigenetic age acceleration is associated with smoking but not AMD in whole blood. EAA analysis was conducted using DNAm microarray data to compare differences from Smoker $(n=19)$ and Non-Smoker $(n=11)$ whole blood gDNA using the (A) Horvath multi-tissue, (B) Hannum and, (C) Skin \& Blood epigenetic clocks. EAA analysis was further stratified to compare differences amongst the following four groups: Normal Non-Smoker $(n=6)$; AMD Non-Smoker $(n=5)$; Normal Smoker $(n=10)$; AMD Smoker $(n=9)$ using (D) Horvath's multi-tissue, (E) Hannum's and (F) the Skin \& Blood epigenetic clocks. Smokers displayed a significant increase in EAA using (A) Horvath's multi-tissue clock $(p=0.0071)$, (B) Hannum's clock $(p=0.0014)$, and $(\mathbf{C})$ the Skin \& Blood clock $(p=0.0025)$ when compared to Non-Smoker groups. Following stratification into four separate groups, no significant differences were observed following one-way ANOVA when analysing (D) Horvath's multi-tissue epigenetic clock, but were identified using the (E) Hannum and (F) Skin \& Blood epigenetic clocks (Supplementary Materials: Additional File S2, Table S5). Following application of the TukeyHSD post-hoc test, no significant differences were identified when comparing Normal Non-Smoker and AMD Non-Smoker, in addition to Normal Smoker and AMD Smoker groups using the (E) Hannum and (F) Skin \& Blood epigenetic clocks. Significant differences were identified when comparing EAA between AMD Non-Smokers and Normal Smokers using the (E) Hannum and (F) Skin \& Blood epigenetic clocks. When comparing AMD Non-Smoker and AMD Smoker groups, no significant difference was found when comparing the (E) Hannum epigenetic clock, however a significant increase in EAA was found using the (F) Skin \& Blood epigenetic clock (for individual p-values, see Supplementary Materials: Additional File S2, Table S6). Statistical analysis was performed using (A-C) an unpaired T-test of means and an (D-F) Ordinary one-way ANOVA followed by the (E,F) TukeyHSD post-hoc test. $\left({ }^{*} p \leq 0.05\right) .\left({ }^{*} p \leq 0.01\right)$.

\subsection{AMD Smokers Display Positive Epigenetic Age Acceleration Using the Skin E Blood Epigenetic Clock}

We next investigated whether presence of AMD was associated with EAA in Smokers and Non-Smokers, and whether smoking status was associated with EAA in AMD or Normal samples using whole blood-derived gDNA. We stratified our analysis to compare the following four groups: Normal Non-Smoker $(n=6)$; AMD Non-Smoker $(n=5)$; Normal Smoker $(n=10)$; and AMD Smoker $(n=9)$, using the Horvath multi-tissue (Figure 3D), Hannum (Figure 3E), and Skin \& Blood (Figure 3F) epigenetic clocks, respectively. Individual $p$-values for each respective comparison across all clocks tested are listed in Supplementary Materials: Additional File S2, Tables S5 and S6.

Firstly, we performed a one-way ANOVA for the Horvath multi-tissue (Figure 3D), Hannum (Figure 3E) and Skin \&Blood (Figure 3F) epigenetic clocks. A Benjamini-Hochberg FDR correction was applied to F-test values for each one-way ANOVA (Supplementary Materials: Additional File S2, Table S5). The Horvath multi-tissue clock did not display significant FDR-adjusted p-values (Figure 3D). However, the Hannum (Figure 3E) and Skin \& Blood (Figure 3F) epigenetic clocks did display significant FDR-adjusted $p$-values and were selected for the TukeyHSD post-hoc test to assess differences across groups. 
Details of the one-way ANOVA with the applied TukeyHSD post-hoc test are found in Additional File S2, Table S6. Significant differences were identified in EAA between AMD Non-Smokers and Normal Smokers using the Hannum ${ }^{*} p=0.0334$, mean year difference: -8.42 years) and Skin \& Blood clocks ( $* * p=0.0064$, mean year difference: -8.94 years). However, we did not observe any differences in EAA between Normal Non-Smoker compared to AMD Non-Smoker; and Normal Smoker compared to AMD Smoker groups using the Hannum (Figure 3E) and Skin \& Blood (Figure 3F) epigenetic clocks, suggesting that disease state is not associated with positive or negative EAA in Smokers. We next sought to assess whether smoking was associated with EAA in Normal and AMD patients. Normal Smoker patients did not display any increase in EAA compared to Normal Nonsmokers. However, in AMD patients, smoking was associated with a significant positive EAA when compared to Non-Smokers in AMD using the Skin \& Blood epigenetic clock ( ${ }^{*} p=0.0335$, mean year difference: -7.347 years). (Figure $\left.3 \mathrm{E}, \mathrm{F}\right)$.

\subsection{Differentially Methylated C $p G$ Probes Identified in Whole Blood gDNA from AMD Patients}

We next investigated DNAm differences at individual CpG probes in AMD $(n=14)$ and Normal $(n=16)$ whole blood-derived gDNA using DNAm data generated by the EPICarray. We conducted differentially methylated locus (DML) and differential region (DMR) analysis between AMD and Normal samples. All AMD samples used in our analysis from the Southampton Cohort were AREDS grade $2(78 \%)$ or grade $3(22 \%)$. No significant CpG probes or regions were identified in our cohort following FDR multiple testing correction in our DML or DMR analysis [32]. However, applying an unadjusted $p$-value cut-off criterion of $p \leq 10^{-4}$ [27], we identified 21 differentially methylated CpG probes between AMD and Normal groups (Table 1). We identified five promoter based $\mathrm{CpG}$ probes, 14 gene body based $\mathrm{CpG}$ probes, and two intergenic probes. Across the top 21 variable probes $\left(p \leq 10^{-4}\right)$, we observed low mean $\beta$-value differences between AMD and Normal samples $(\Delta \beta)(\Delta \beta \pm \leq 5 \%)$ with two CpG probes displaying large effect sizes $(\Delta \beta \pm \geq 10 \%)$. The largest effect size was identified in RPTOR (cg04953735, Intron $3, \Delta \beta=-11.95 \%)$. For a scatter plot representing methylation $\beta$-values, please see Supplementary Materials: Additional File S2, Figure S1. cg04953735 within RPTOR co-localised with H3K4me1 and H3K27ac enrichment using layered data in seven cell lines from ENCODE, UCSC genome browser, suggestive of methylation differences occurring within an active enhancer region. In addition, cg04953735 lies within a predicted 2032bp enhancer region (GeneHancer: GH17J080678) within intron 3 of RPTOR, 134008bp upstream of a predicted interaction region within the TSS200 of RPTOR. 
Table 1. Differentially methylated CpG probes identified in whole blood of patients with AMD using a relaxed $p$-value criterion $\left(p \leq 10^{-4}\right)$.

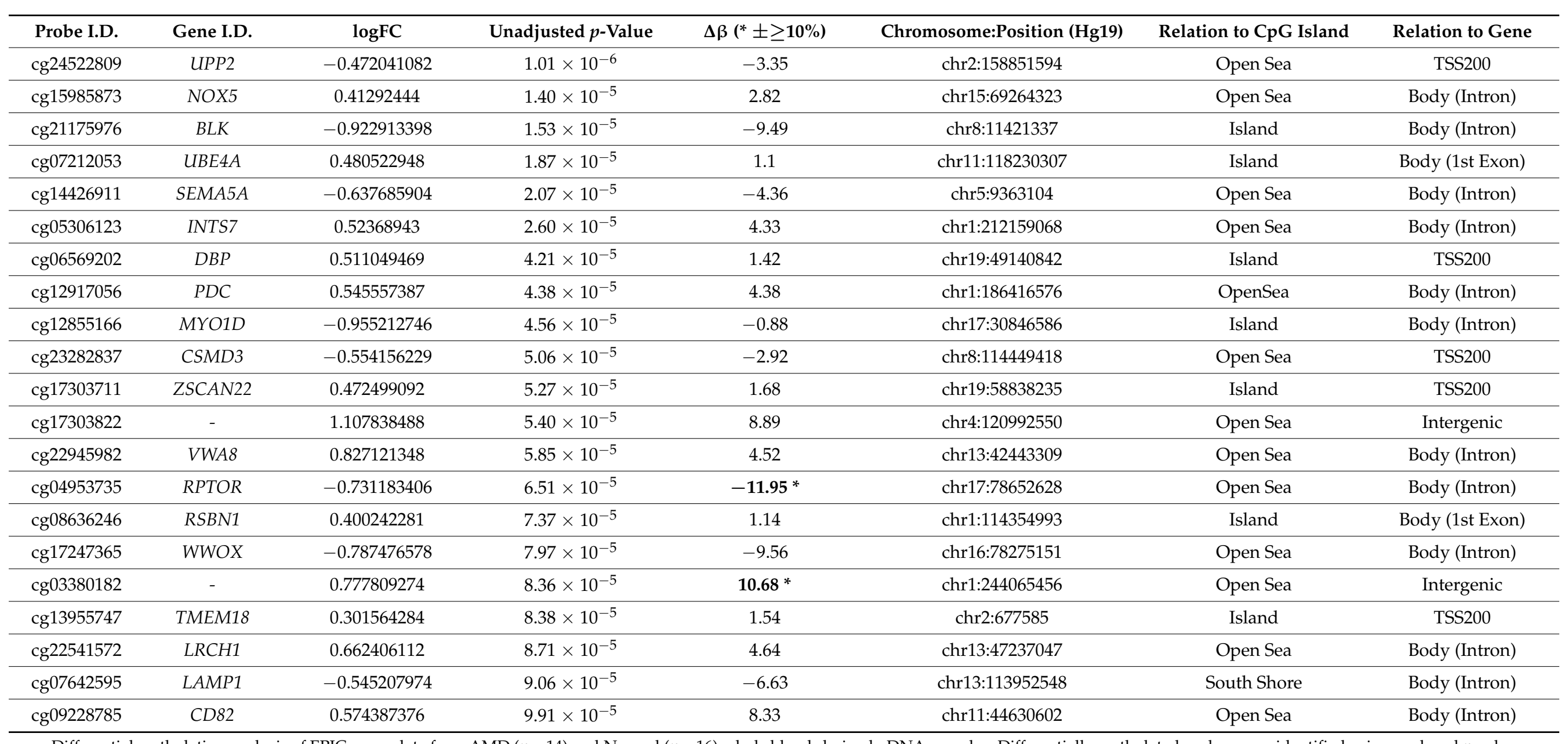

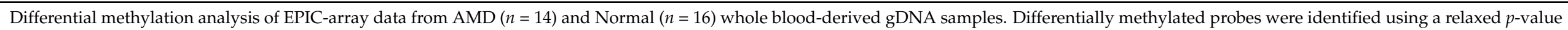

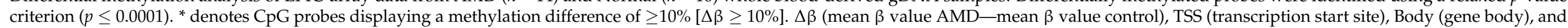
Intergenic (Intergenic region not mapping to known gene). 


\subsection{Ingenuity Pathway Causal Network Analysis Identified RPTOR as a Master Regulator of Methylation Changes in RPE}

We performed moderated $t$-tests using limma and conducted IPA CNA on the top 301 most significantly differentially methylated CpG probes in blood, based on a $p$-value threshold of $p \leq 10^{-3}$ between AMD and Normal groups. Following filtering of CpG probes in intergenic areas and assigning multiple $\mathrm{CpG}$ probes mapping to a single gene as one single gene, the IPA CNA in blood revealed 212 genes for analysis. Supplementary Materials: Additional File S3, demonstrates the full results of IPA CNA for the top most differentially methylated $\mathrm{CpG}$ probes between AMD and Normal samples in blood. We also assessed the top most variable probes from our published genome wide DNA methylation dataset obtained from RPE [27]. We performed IPA CNA by scoring against the disease terms "macular degeneration" (Figure 4). No master regulators were identified among the top 301 most significantly differentially methylated probes $\left(p \leq 10^{-3}\right)$ using whole blood-derived gDNA from the Southampton Cohort that scored against the term "macular degeneration" (Additional File S3). However, in the RPE, IPA CNA identified inhibition of RPTOR (Figure 4) as master regulator for methylation changes amongst the top most significantly differentially methylated CpG probes that scored against the term "macular degeneration" in AMD RPE [27]. Full results of IPA CNA for the top most significantly differentially methylated CpG probes between AMD and Normal samples in RPE, are shown in Supplementary Materials: Additional File S3. In addition, the predicted inhibition of RPTOR in AMD RPE leads to the predicted inhibition of CTNNB1 encoding beta-catenin, predicted to activate the disease term "macular degeneration".

A

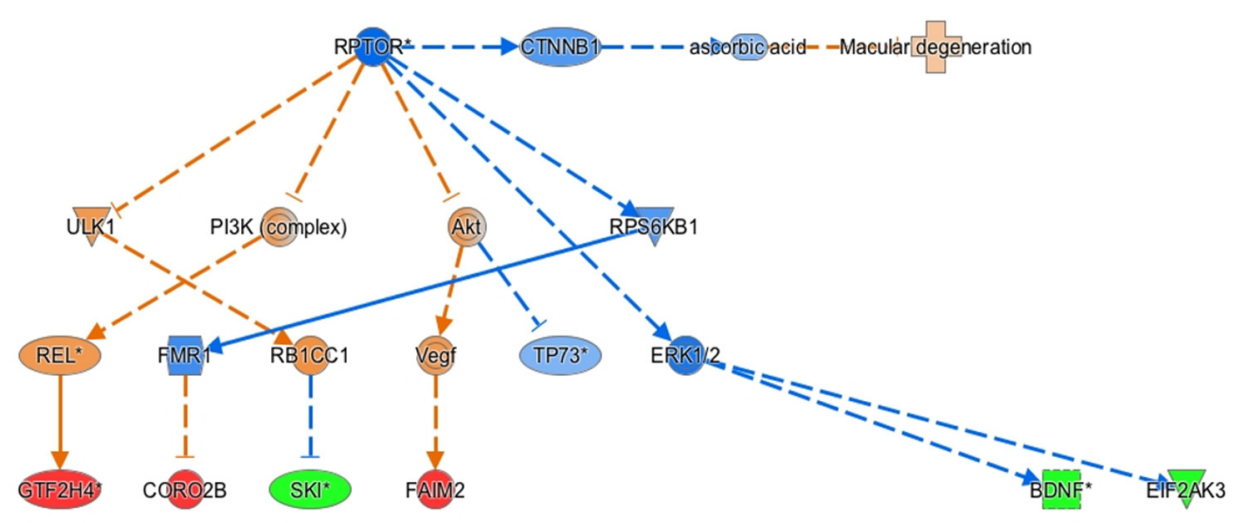

B

Figure 4. Ingenuity Pathway Analysis Causal Network Analysis identifies RPTOR as a master regulator for DNA methylation changes in AMD RPE. (A) Ingenuity Pathway Analysis Causal Network Analysis was performed on the top most differentially methylated probes identified by the 450K-array in AMD human RPE gDNA samples. RPTOR inhibition was identified as a master upstream regulator of SKI, GTF2H4, CORO2B, FAIM2, BDNF, and EIF2AK3. RPTOR inhibition was also found to inhibit CTNNB1, which inhibits ascorbic acid, predicted to activate the term "macular degeneration" in IPA. (B) Prediction legend for the Ingenuity Pathway Analysis Causal Network Analysis.

\section{Discussion}

This is the first study to our knowledge formally evaluating whether EAA is associated with AMD and important risk factor covariates including smoking status. We sought to address whether EAA is observed in the RPE [27], as it is a primary site of AMD pathogenesis, and in whole blood, as the epigenetic clocks have been widely applied and validated in blood-derived gDNA.

In the RPE, we observed a marked negative EAA across all groups with no significant differences in EAA between AMD and Normal samples using all three clocks. This result cannot be characterised as true negative age acceleration because of poor performance 
of the epigenetic clocks in RPE [33]. The consistent poor correlation of predicted DNAm age with chronological age observed in the RPE markedly improved when analysing whole blood-derived gDNA data, explained by the datasets used to train each respective epigenetic clock $[3,4,8]$. Although we might expect any non-zero EAA in the RPE to also be associated with weak correlation, our findings are key in illustrating the potential biological and technical deviations present when assaying RPE samples as opposed to whole blood using the epigenetic clocks. Non-zero deviations do indeed have an impact on the accuracy and correlation of the estimates; however, we posit that the epigenetic age is always underestimated in RPE rather than errors being positively and negatively distributed around the mean. This suggests clock calibration issues in RPE or a currently unknown biological phenomenon, although the better predictive capacity of the clocks in blood supports the former assumption. As such, we believe the poor accuracy of epigenetic clocks in RPE is a key finding, especially in the context of age-dependent hypermethylation of ELOVL2 in ageing ocular tissues increasingly referenced as a reliable biomarker $[4,8,34]$. The data provided welcomes the generation of multivariate models to further delineate the complexity of ageing in RPE or address the current calibration issues with existing clocks.

Reasonable performance of each respective epigenetic clock in whole blood, however, strengthens the observation of no association of EAA with AMD in blood, though this remains open to further investigation in the RPE, which can be addressed using a bespoke RPE epigenetic clock with greater predictive accuracy. The highly accurate skeletal muscle [35] and breast tissue [36] epigenetic clocks have set precedents to this approach. These clocks share little overlap in CpG probe selection with Horvath's multi-tissue clock suggesting that bespoke clocks capture separate tissue specific epigenetic ageing processes [36]. Therefore, construction of a tissue-specific RPE clock is necessary for future studies to capture the specific epigenetic ageing processes in the RPE.

A strength of this study was having information regarding the smoking status of AMD patients from which blood samples were taken, allowing stratified analyses to investigate whether smoking was associated with EAA in AMD. Smoking, the greatest modifiable risk factor for AMD, substantially increases AMD relative risk (RR) with RR of disease varying from 2.7 to 6.6 in current smokers in unrelated mixed-gender cohorts [19-22]. In accordance with previous studies examining EAA using Horvath's multi-tissue clock on DNAm data from lung tissue of smokers [30], we observed a significant positive EAA in all three epigenetic clocks tested. The three clocks are predictors of chronological ageing and display a degree of $\mathrm{CpG}$ probe overlap [6], however each clock captures facets of a diverse, often tissue-type specific ageing process [6]. Taken together, our results suggest that smoking exerts a significant and broad effect on DNAm age in blood and further regulates biological ageing across several tissue types. To confirm whether this effect is present in the RPE, future studies will require smoking status documentation.

Using the Skin \& Blood clock, which demonstrated the greatest predictive accuracy for chronological age in our whole blood samples, we found significant positive EAA in AMD Smokers compared to Non-Smokers, a finding not replicated in the Horvath multi-tissue [3] and Hannum epigenetic clocks [4]. One explanation for this may lie in confounding factors affecting clock performance based on the age range of patient samples used in our study, which included RPE/blood from patients $>50$ years old. Previous studies have demonstrated failure of both the Horvath multi-tissue and Hannum epigenetic clocks to accurately predict chronological age in older cohorts [12,33,37]. It is proposed that this occurs due to methylation saturation (i.e., select $\mathrm{CpG}$ probes reaching either 0 or $100 \%$ methylation later in life), in addition to confounding from other age related processes [33]. In the case of Horvath's multi-tissue epigenetic clock, poor predictive capability in elderly samples can be partially attributed to lower representation of tissues from elderly individuals in test data [33]. Inaccurate prediction of DNAm age in elderly individuals has also been shown to be a facet of Hannum's clock [33], where test data individuals were aged between 19-101 years [4]. Therefore, the reasons for less precise age prediction in older individuals using the Hannum/Horvath clock are not entirely clear. 
However, previous poor performance in older datasets for the Horvath multi-tissue and Hannum clocks may explain why the Skin \& Blood clock performed best out of all three clocks tested in blood-derived gDNA. While the Skin and Blood clock has also shown a degree of error in elderly samples due to age confounding [37], it consistently outperforms predictive capacity in blood samples when compared to Horvath's multi tissue clock and Hannum's clock, a finding confirmed in this study $[7,37]$. Therefore, our observation that smokers with AMD exhibit accelerated epigenetic ageing compared to Non-Smokers with AMD is strengthened by identifying this effect in the clock with the greatest predictive accuracy for the demographic age and tissue type represented in our dataset.

To support our analyses of epigenetic ageing in AMD, we performed DML analysis in AMD whole blood-derived gDNA. In line with previous microarray-based studies in blood of patients with AMD [26], we were unable to identify significantly differentially methylated CpG probes following FDR-adjusted multiple testing correction. However, using a $p$-value cut-off criterion of $p \leq 10^{-4}$ [27], we identified 21 differentially methylated CpG probes, with no overlap between our top 21 most variable probes and those observed in other genome-wide studies [26,27]. Small effect size was observed amongst our top most variable probes $(\Delta \beta \leq 5 \%)$, consistent with the effect sizes reported in the literature $[23,26]$. We propose that this effect is similar to the ageing process outlined by Horvath's multitissue clock, whereby DNAm levels at individual CPG probes correlate poorly with age, but the composite effect of a larger number of probes represents consistent and reproducible epigenetic changes occurring during the ageing process [3]. Collectively, our finding suggests that a composite effect of small methylation changes at a number of $\mathrm{CpG}$ probes drives early and intermediate AMD, representing a more accurate picture of DNAm changes occurring during AMD development $[3,38]$.

We next investigated a causal relationship for methylation changes using IPA CNA at the top 301 most significantly differentially methylated $\mathrm{CpG}$ probes in blood and top significantly differentially methylated $\mathrm{CPG}$ probes in the RPE, identified in our previous study [27]. IPA CNA may detect novel master upstream regulators acting through intermediate downstream regulators affecting gene expression [39], in this case using directional log-fold methylation changes as a proxy for expression changes. IPA CNA creates networks based on known interactions within the IPA knowledge base [39]. While the causal relationship of methylation changes between genes is less defined than that of gene expression differences, IPA CNA can be used to identify master regulators [39]. IPA CNA maps differentially methylated probes to genes, and then maps these genes to disease states, such as AMD. However, previous studies identified bias in gene-set analysis applied to high throughput DNAm data [40]. The reason for this is the key assumption in GSA that genes have, a priori, the same probability of appearing in the list regardless of experimental condition [40]. In the case of DNAm data, this can lead to bias due to differences in the prevalence of $\mathrm{CpG}$ probes associated with different genes [40]. IPA accounts for this bias by recognising a gene that appears multiple times in a list only once. In this context, we were unable to identify master regulators of genes of differentially methylated $\mathrm{CpG}$ probes in our top 301 significantly differentially methylated $\mathrm{CpG}$ probes in whole blood, however we identified inhibition of RPTOR as the putative upstream master regulator in AMD RPE [27].

Importantly, in blood, we also identified the largest methylation change of any probe within RPTOR, with a substantial decrease in methylation $\left(\Delta \beta=-11.5 \%\right.$; $\left.p=6.51 \times 10^{-5}\right)$. Raptor is an adaptor protein involved in regulating the activity of Mammalian Target of Rapamycin Complex-1 (mTORC1), by facilitating recruitment of substrates to the mTOR kinase [41] which regulates ageing, cellular growth, stress responses and inhibits autophagy [42]. mTOR plays a central role in ageing and is implicated in proteostasis, mitochondrial function and cellular senescence [43]. Raptor inhibition has been shown in human donor AMD RPE concurrent with increased mTOR activation [42] a finding corroborated by previous evidence showing that primary RPE cell cultures from elderly individuals display increased mTORC1 activity [44]. Of further relevance, overactive mTORC1 has 
been shown to indirectly downregulate the expression of protein kinase ER-like Kinase (PERK), a key component of the unfolded protein response, in mouse embryonic fibroblasts [45]. EIF2AK3, the gene encoding PERK, displays significant downregulation in AMD RPE [27]. Together, these findings support key roles for upregulated mTORC1 activity and inhibition of RPTOR in AMD, although further experimental evidence is required to confirm this association.

We acknowledge that whilst there is added utility in the assessment of epigenetic ageing in primary affected disease tissues such as RPE in AMD, epigenetic ageing processes in peripheral tissues, such as blood, may still provide useful insights to generate a systemic ageing profile associated with AMD. In this context, evidence suggests that although AMD is primarily an ocular disease, systemic factors contribute to disease development $[26,46]$. The growing body of evidence identifying DNAm changes both in RPE [27] and in blood [26] of AMD patients is important as altered epigenetic maintenance processes implicated in ageing and ageing-related diseases, may potentially be reversed. Lu et al. recently demonstrated that epigenetic ageing is not a unidirectional process, as induced ectopic expression of the OCT4, SOX2 and KLF4 genes in mouse retinal ganglion cells reversed vision loss in mouse models of glaucoma and ageing, associated with restoration of a youthful DNAm age [47].

A limitation of this study lies in the sample size used to investigate differences in methylation between AMD and Normal whole blood-derived gDNA with insufficient power to detect FDR-adjusted differentially methylated CpG probes. This may also underlie the absence of EAA associated in AMD. However, a previous EWAS investigating DNAm differences in whole blood failed to identify significant differential methylation following FDR-adjustment using a significantly increased cohort size (AMD, $n=198$; Control, $n=100$ ) [26]. Therefore, future studies investigating both differential methylation and EAA will require greater samples sizes [26,27].

In conclusion, our findings of positive EAA using all three epigenetic clocks in Smokers, and in Smokers with AMD using the most appropriate Skin \& Blood epigenetic clock represents an important avenue for further development [47]. Furthermore, our identification of RAPTOR as master regulator of DNAm changes in AMD RPE supports its role in both ageing and AMD and provides a key target for future functional studies.

\section{Materials and Methods}

\subsection{Sample Collection, Grading and DNA Extraction}

Peripheral whole blood samples and gDNA were extracted from individuals within the Southampton Case-Control Cohort (referred to as the "Southampton Cohort") in a manner described previously [48]. Demographic information was provided including age (years), gender (male or female), and smoking status (Smoker or Non-Smoker) (Supplementary Materials: Additional File S2, Table S1). Age-matching statistics for the purpose of this study comparing AMD and Normal groups, in addition to Non-Smoker and Smoker groups, can be found in Supplementary Materials: Additional File S2, Tables S3 and S4. Samples were obtained from individuals phenotyped according to the Age-Related Eye Disease Study (AREDS) classification (Additional File S2: Table S2) [49]. Samples from patients exhibiting advanced AMD were excluded from the study. A total of 30 patient samples including 16 Normal, 3 AREDS grade 2 (early AMD) and 11 AREDS grade 3 (intermediate AMD) (AMD total, $n=14$ ) were selected (Supplementary Materials: Additional File S2, Table S1). Analysis of peripheral whole blood gDNA degradation levels were assessed prior to bisulfite conversion for methylation analyses by performing gel electrophoresis in a previously described manner [27].

\subsection{Illumina Infinum MethylationEPIC BeadChip Array}

DNAm levels were measured using the EPIC-array (Illumina Inc., San Diego, CA, USA), interrogating $865918 \mathrm{CpG}$ sites covering $\geq 99 \%$ of RefSeq genes. Samples run on the EPIC-array were randomized and balanced for disease status and smoking status to 
minimise chip and row specific effects. The EPIC-array was conducted at the Edinburgh Clinical Research Facility (Edinburgh, UK) incorporating technical controls into the experimental design. In total, $500 \mathrm{ng}(50 \mathrm{ng} / \mu \mathrm{L})$ peripheral whole blood-derived gDNA was bisulfite converted using the EZ-96 DNA methylation kit (Zymo Research, Irvine, CA, USA) and hybridised to the EPIC-array according to the manufacturer's instructions. Quality control analysis was performed using GenomeStudio (v2011.1). Raw IDAT files were then read into $R$ (version 3.31) using the read.metharray.exp function within the minfi package [50] The dataset and analysis of the Southampton Cohort has been deposited in ArrayExpress with submission number: E-MTAB-11279.

\subsection{Pre-Processing and Normalisation}

EPIC-array data was analysed using functionality within the minfi package [50]. Each sample was subjected to various quality control measures. Cell type proportions were corrected using the estimateCellCounts2 function in R. Briefly, "CD8T", "CD4T", "NK", "Bcell", "Mono", and "Neu" were deconvoluted from our mixed whole blood samples [51]. Furthermore, samples were checked for global hybridisation quality based on an average probe detection $p$-value threshold of $p \geq 0.05$. Samples not meeting these criteria were removed from the analysis. Individual CpG probes exceeding a detection $p$-value of $p \geq 0.01$, indicating a failed position, were removed from the analysis. Probes located on chromosomes $\mathrm{X} \& \mathrm{Y}$ and probes within two base pairs of a single nucleotide polymorphism (SNP) with a minor allele frequency $\geq 0.05$ were also removed, as well as probes previously found to cross hybridise to multiple genomic locations [52,53]. Samples were normalised using the Subset-quantile Within Array Normalisation (SWAN) algorithm to correct for biases between type I and type II probe distributions [54]. Following filtering, 784,486 CpG probes were available for downstream analysis.

\subsection{Epigenetic Clock Analyses}

DNAm age was calculated on DNAm data obtained from whole blood-derived gDNA of Normal and AMD patients from the Southampton Cohort, analysed using the EPICarray, and DNAm data from ocular tissue (human RPE) of Normal and AMD patients from our previously described cohort of individuals of European descent, analysed using 450K-array data (accessed from ArrayExpress: E-MTAB-7183) [date accessed: 24 May 2020]. [27]. DNAm age estimations were performed using Horvath's multi-tissue [3], Hannum's [4], and the Skin \& Blood epigenetic [8] clocks using a publicly available online calculator (available at: http://dnamage.genetics.ucla.edu/, accessed on 9 September 2021). For outputs of all epigenetic clock data in whole blood and RPE-derived gDNA, please see Supplementary Materials: Additional File S1. We assessed the performance of each respective clock on DNAm data derived from RPE gDNA $(n=44)$ using 450K-array data from our previously described cohort [27]. We also assessed the performance of these three clocks using whole blood-derived $(n=30)$ gDNA methylation data acquired by EPIC-array from the Southampton Cohort. Performance was assessed using linear regression of estimated DNAm age with chronological age of patient-derived samples from each respective tissue type. EAA was calculated as the difference between estimated DNAm age and chronological age (DNAm age-chronological age). Assessment for normal distribution of data was performed using a Shapiro-Wilks Test for Normality prior to conducting comparisons using statistical tests. Normality tests were passed following $\alpha>0.05$. Non-parametric Mann-Whitney tests comparing EAA of whole blood-derived gDNA samples and RPE-derived gDNA samples were performed across the groups: "AMD vs Normal" in whole blood and RPE. Univariate analyses employing an unpaired $t$-test comparing EAA was performed for: "Smoker vs Non-Smoker" groups. We stratified our cohort into the following groups: Normal Non-Smoker $(n=6)$; AMD Non-Smoker $(n=5)$; Normal Smoker $(n=10)$; and AMD Smoker $(n=9)$. We computed ANOVAs for each respective epigenetic clock. We applied the FDR correction to the $F$ Test values of each ANOVA and, if significant following FDR adjustment, applied the TukeyHSD post-hoc test 
to determine significant differences between groups (Supplementary Materials: Additional File S2, Tables S5 and S6). All statistical analysis was performed using $R$ (version 3.31) and GraphPad Prism (ver 8.0.2, GraphPhad Software Inc., San Diego, CA, USA).

\subsection{Differentially Methylated CpG Probe and Region Analysis}

To identify differentially methylated CpG probes, univariate statistical analysis for each CpG probe was performed using the linear models for microarray data (Limma) package [55]. To implement the linear models, we built a contrast matrix with coefficients for "AMD" and "Normal". Significance was determined based on a Benjamini-Hochberg $(\mathrm{BH})$-adjusted False Discovery Rate $(\mathrm{FDR}) \leq 0.05$. In a scenario where no differentially methylated $\mathrm{CpG}$ probes were found using a BH-adjusted FDR $\leq 0.05$, an unadjusted $p$-value criterion of $p \leq 10^{-4}$ was applied [27]. We assessed for differentially methylated regions using DMRcate [56]. Regions were defined as blocks of 1000 nucleotides fitting a gaussian kernel smoothed function. We considered a region to be differentially methylated if its $\mathrm{BH}$-corrected FDR was $\leq 0.1$ and had an absolute mean beta fold change $>0.1(10 \%)$ ).

\subsection{Histone Modification Enrichment and GeneHancer Analysis}

For the top most variable probes in whole blood DNAm data meeting the unadjusted $p$-value criterion of $p \leq 10^{-4}$, analysis of histone modification enrichment was performed using the overlaid $\mathrm{H} 3 \mathrm{~K} 4 \mathrm{Me} 1, \mathrm{H} 3 \mathrm{~K} 4 \mathrm{Me}$, and $\mathrm{H} 3 \mathrm{~K} 27 \mathrm{Ac}$ track functions within the Integrated Regulation track from the UCSC genome browser (available from: https: / / genome.ucsc.edu/cgi-bin/hgTrackUi?g=wgEncodeReg, accessed on 5 August 2021). The layered H3K4Me1, H3K4Me3, and H3K27Ac tracks consist of Chromatin Immunoprecipitation Sequencing data from seven cell lines: GM12878, H1-hESC, HSMM, HUVEC, K562, NHEK, and NHLF. GeneHancer analysis was performed using the Enhancer and Promoter functions from GeneHancer track within the UCSC genome browser (available from: https: / / genome.ucsc.edu/cgi-bin/hgTrackUi?db=hg19\&g=geneHancer, accessed on 5 August 2021) to identify putative enhancer and promoter elements signatures. Additionally, interactions between predicted enhancers and regions within target genes were analysed using the Interaction function within the Enhancer and Promoter from GeneHancer track.

\subsection{Ingenuity Pathway Analysis}

The Causal Network Analysis (CNA) function within Ingenuity Pathway Analysis (IPA) was used to construct networks to investigate novel upstream regulators associated with AMD. Both direct and indirect relationships were considered in the general settings. All node types and data sources were considered, and confidence gained using experimentally observed and high (predicted) interactions. Interactions were considered from available mammals (human, mouse, and rat). All tissue lines and mutations were considered. CNA was performed against the disease term "macular degeneration" for the variable probes from both respective datasets (RPE and blood) to identify master regulators based on activation $z$-scores $(z \geq 2.0)$. This was performed on the top most differentially methylated probes that met the $p$-value criterion $\left(p \leq 10^{-6}\right)$ in the RPE of patients with AMD, as published in [27] (Additional File S3); in addition to the top-301 variable probes $\left(p \leq 10^{-3}\right)$ identified in blood-derived gDNA in patients with AMD (Additional File S3).

Supplementary Materials: The following are available online at https:/ / www.mdpi.com/article/10 $.3390 /$ ijms222413457/s1.

Author Contributions: L.F.P. proposed the project. L.F.P., L.P. and N.S. developed the concept. L.F.P., N.S. and D.G. conceived analysis required. A.L. and A.C. collected the Southampton AMD cohort. N.S. and D.G. performed the data analysis. L.F.P., N.S. and D.G. wrote the manuscript. N.S., D.G., A.L., L.F.P. and L.P. revised the manuscript. L.F.P. and L.P. supervised this work. All authors have read and agreed to the published version of the manuscript. 
Funding: L.F.P. is MCU-PH funded by the French Ministry of Health at the University of Strasbourg, France; previously an NIHR Clinical Lecturer at the University of Liverpool. This project was funded by Academy of Medical Sciences Starter Grant for Clinical Lecturers awarded to L.F.P. N.S. is supported by a National Eye Research Centre PhD Studentship. D.G. is funded by the MRC Versus Arthritis Centre for Integrated Research into Musculoskeletal Ageing (MR/R502182/1). The views expressed are those of the author(s) and not necessarily those of the NHS, the NIHR, or the Department of Health. The funders had no role in the design, data collection, analysis, or conclusions drawn from this study. AJL is an emeritus NIHR Senior Investigator and the Southampton AMD cohort was funded by Macula Vision Research Foundation, Macular Disease Society, British Council Prevention Blindness, T.F.C. Frost Charitable Trust, The Wellcome Trust, Brian Mercer Charitable Trust, De Laszlo Foundation and the Gift of Sight Appeal.

Institutional Review Board Statement: The study was conducted according to the guidelines of the Declaration of Helsinki, and approved by the Institutional Review Board (or Ethics Committee) of the University of Liverpool Central University Research Ethics Committee for Physical Interventions, reference 2326; date of approval 31 January 2018.

Informed Consent Statement: Informed written consent was obtained from individuals who donated their blood samples for the purposes of this research.

Data Availability Statement: All datasets utilised here are open-access datasets accessible on ArrayExpress and listed in Section 4.

Acknowledgments: We thank Nicola Wrobel of the Edinburgh Clinical Research Facility Genetics Core for processing the methylation arrays. We thank Helen Griffiths for DNA extraction of the Southampton samples. We thank Venkata Murthy Chavali for critical review of the manuscript.

Conflicts of Interest: The authors declare no conflict of interest.

$\begin{array}{ll}\text { Abbreviations } \\ \text { 450K-array } & \text { Illumina Infinium HumanMethylation450 BeadChip Array } \\ \text { AREDS } & \text { Age-related Eye Disease Study } \\ \text { AMD } & \text { Age-related Macular Degeneration } \\ \text { DNAm } & \text { DNA methylation } \\ \text { DNAm Age } & \text { DNA methylation age } \\ \text { EAA } & \text { Epigenetic Age Acceleration } \\ \text { EMS } & \text { Epigenetic Maintenance System } \\ \text { EPIC-array } & \text { Illumina Infinium MethylationEPIC BeadChip Array } \\ \text { FDR } & \text { False Discovery Rate } \\ \text { gDNA } & \text { Genomic DNA } \\ \text { IPA CNA } & \text { Ingenuity Pathway Analysis; Causal Network Analysis } \\ \text { mTORC1 } & \text { Mammalian Target of Rapamycin Complex 1 } \\ \text { PERK } & \text { Protein Kinase ER-like Kinase } \\ \text { RAPTOR } & \text { Regulatory Associated Protein of mTOR Complex 1 } \\ \text { RPE } & \text { Retinal Pigment Epithelium } \\ \text { SWAN } & \text { Subset-quantile Within Array Normalisation }\end{array}$

\section{References}

1. Niccoli, T.; Partridge, L. Ageing as a Risk Factor for Disease. Curr. Biol. 2012, 22, R741-R752. [CrossRef] [PubMed]

2. Jung, M.; Pfeifer, G.P. Aging and DNA methylation. BMC Biol. 2015, 13, 7. [CrossRef]

3. Horvath, S. DNA methylation age of human tissues and cell types. Genome Biol. 2013, 14, R115. [CrossRef]

4. Hannum, G.; Guinney, J.; Zhao, L.; Zhang, L.; Hughes, G.; Sadda, S.; Klotzle, B.; Bibikova, M.; Fan, J.-B.; Gao, Y.; et al. Genome-wide Methylation Profiles Reveal Quantitative Views of Human Aging Rates. Mol. Cell 2012, 49, 359-367. [CrossRef] [PubMed]

5. Levine, M.E.; Lu, A.T.; Quach, A.; Chen, B.H.; Assimes, T.L.; Bandinelli, S.; Hou, L.; Baccarelli, A.A.; Stewart, J.D.; Li, Y.; et al. An epigenetic biomarker of aging for lifespan and healthspan. Aging 2018, 10, 573-591. [CrossRef]

6. Bergsma, T.; Rogaeva, E. DNA Methylation Clocks and Their Predictive Capacity for Aging Phenotypes and Healthspan. Neurosci. Insights 2020, 15, 2633105520942221. [CrossRef] [PubMed]

7. Horvath, S.; Raj, K. DNA methylation-based biomarkers and the epigenetic clock theory of ageing. Nat. Rev. Genet. 2018, 19, 371-384. [CrossRef] 
8. Horvath, S.; Oshima, J.; Martin, G.M.; Lu, A.T.; Quach, A.; Cohen, H.; Felton, S.; Matsuyama, M.; Lowe, D.; Kabacik, S.; et al. Epigenetic clock for skin and blood cells applied to Hutchinson Gilford Progeria Syndrome and ex vivo studies. Aging 2018, 10, 1758-1775. [CrossRef]

9. Levine, M.E.; Lu, A.T.; Bennett, D.A.; Horvath, S. Epigenetic age of the pre-frontal cortex is associated with neuritic plaques, amyloid load, and Alzheimer's disease related cognitive functioning. Aging 2015, 7, 1198-1211. [CrossRef]

10. Horvath, S.; Ritz, B.R. Increased epigenetic age and granulocyte counts in the blood of Parkinson's disease patients. Aging 2015, 7, 1130-1142. [CrossRef]

11. Xiao, C.; Beitler, J.J.; Peng, G.; Levine, M.E.; Conneely, K.N.; Zhao, H.; Felger, J.C.; Bs, E.C.W.; Bs, C.E.C.; Jeon, S.; et al. Epigenetic age acceleration, fatigue, and inflammation in patients undergoing radiation therapy for head and neck cancer: A longitudinal study. Cancer 2021, 127, 3361-3371. [CrossRef]

12. Horvath, S.; Mah, V.; Lu, A.T.; Woo, J.S.; Choi, O.-W.; Jasinska, A.J.; Riancho, J.A.; Tung, S.; Coles, N.S.; Braun, J.; et al. The cerebellum ages slowly according to the epigenetic clock. Aging 2015, 7, 294-306. [CrossRef] [PubMed]

13. Zheng, C.; Li, L.; Xu, R. Association of Epigenetic Clock with Consensus Molecular Subtypes and Overall Survival of Colorectal Cancer. Cancer Epidemiol. Biomark. Prev. 2019, 28, 1720-1724. [CrossRef] [PubMed]

14. Wang, T.; Maden, S.; Luebeck, G.E.; Li, C.I.; Newcomb, P.A.; Ulrich, C.M.; Joo, J.-H.E.; Buchanan, D.D.; Milne, R.L.; Southey, M.C.; et al. Dysfunctional epigenetic aging of the normal colon and colorectal cancer risk. Clin. Epigenet. 2020, 12, 5. [CrossRef]

15. Ren, J.-T.; Wang, M.-X.; Su, Y.; Tang, L.-Y.; Ren, Z.-F. Decelerated DNA methylation age predicts poor prognosis of breast cancer. BMC Cancer 2018, 18, 989. [CrossRef] [PubMed]

16. Mitchell, P.; Liew, G.; Gopinath, B.; Wong, T.Y. Age-related macular degeneration. Lancet 2018, 392, 1147-1159. [CrossRef]

17. Bourne, R.; Steinmetz, J.D.; Flaxman, S.; Briant, P.S.; Taylor, H.R.; Resnikoff, S.; Casson, R.J.; Abdoli, A.; Abu-Gharbieh, E.; Afshin, A.; et al. Trends in prevalence of blindness and distance and near vision impairment over 30 years: An analysis for the Global Burden of Disease Study. Lancet Glob. Heath 2021, 9, e130-e143. [CrossRef]

18. Wong, W.L.; Su, X.; Li, X.; Cheung, C.M.G.; Klein, R.; Cheng, C.Y.; Wong, T.Y. Global prevalence of age-related macular degeneration and disease burden projection for 2020 and 2040: A systematic review and meta-analysis. Lancet Glob. Health 2014, 2, e106-e116. [CrossRef]

19. Khan, J.C.; Thurlby, D.A.; Shahid, H.; Clayton, D.; Yates, J.R.W.; Bradley, M.M.; Moore, A.T.; Bird, A.C. Smoking and age related macular degeneration: The number of pack years of cigarette smoking is a major determinant of risk for both geographic atrophy and choroidal neovascularisation. Br. J. Ophthalmol. 2006, 90, 75-80. [CrossRef] [PubMed]

20. Tan, J.S.L.; Mitchell, P.; Kifley, A.; Flood, V.; Smith, W.; Wang, J.J. Smoking and the Long-term Incidence of Age-Related Macular Degeneration: The blue mountains eye study. Arch. Ophthalmol. 2007, 125, 1089-1095. [CrossRef]

21. Vingerling, J.R.; Hofman, A.; Grobbee, D.E.; De Jong, P.T.V.M. Age-Related Macular Degeneration and Smoking: The Rotterdam study. Arch. Ophthalmol. 1996, 114, 1193-1196. [CrossRef]

22. Klein, R.; Klein, B.E.K.; Moss, S.E. Relation of Smoking to the Incidence of Age-related Maculopathy: The Beaver Dam Eye Study. Am. J. Epidemiol. 1998, 147, 103-110. [CrossRef] [PubMed]

23. Hunter, A.; Spechler, P.A.; Cwanger, A.; Song, Y.; Zhang, Z.; Ying, G.-S.; Hunter, A.K.; Dezoeten, E.; Dunaief, J.L. DNA Methylation Is Associated with Altered Gene Expression in AMD. Investig. Opthalmol. Vis. Sci. 2012, 53, 2089-2105. [CrossRef]

24. Wei, L.; Liu, B.; Tuo, J.; Shen, D.; Chen, P.; Li, Z.; Liu, X.; Ni, J.; Dagur, P.; Sen, H.N.; et al. Hypomethylation of the IL17RC Promoter Associates with Age-Related Macular Degeneration. Cell Rep. 2012, 2, 1151-1158. [CrossRef]

25. Oliver, V.F.; Franchina, M.; Jaffe, A.E.; Branham, K.E.; Othman, M.; Heckenlively, J.R.; Swaroop, A.; Campochiaro, B.; Vote, B.J.; Craig, J.E.; et al. Hypomethylation of the IL17RC Promoter in Peripheral Blood Leukocytes Is Not A Hallmark of Age-Related Macular Degeneration. Cell Rep. 2013, 5, 1527-1535. [CrossRef]

26. Oliver, V.F.; Jaffe, A.; Song, J.; Wang, G.; Zhang, P.; Branham, K.; Swaroop, A.; Eberhart, C.G.; Zack, D.J.; Qian, J.; et al. Differential DNA methylation identified in the blood and retina of AMD patients. Epigenetics 2015, 10, 698-707. [CrossRef] [PubMed]

27. Porter, L.F.; Saptarshi, N.; Fang, Y.; Rathi, S.; Hollander, A.I.D.; De Jong, E.K.; Clark, S.J.; Bishop, P.N.; Olsen, T.W.; Liloglou, T.; et al. Whole-genome methylation profiling of the retinal pigment epithelium of individuals with age-related macular degeneration reveals differential methylation of the SKI, GTF2H4, and TNXB genes. Clin. Epigenet. 2019, 11, 6. [CrossRef]

28. Kõks, G.; Uudelepp, M.-L.; Limbach, M.; Peterson, P.; Reimann, E.; Kõks, S. Smoking-Induced Expression of the GPR15 Gene Indicates Its Potential Role in Chronic Inflammatory Pathologies. Am. J. Pathol. 2015, 185, 2898-2906. [CrossRef]

29. Ambatipudi, S.; Cuenin, C.; Hernandez-Vargas, H.; Ghantous, A.; Le Calvez-Kelm, F.; Kaaks, R.; Barrdahl, M.; Boeing, H.; Aleksandrova, K.; Trichopoulou, A.; et al. Tobacco smoking-associated genome-wide DNA methylation changes in the EPIC study. Epigenomics 2016, 8, 599-618. [CrossRef] [PubMed]

30. Wu, X.; Huang, Q.; Javed, R.; Zhong, J.; Gao, H.; Liang, H. Effect of tobacco smoking on the epigenetic age of human respiratory organs. Clin. Epigenet. 2019, 11, 183. [CrossRef]

31. Hewitt, A.W.; Januar, V.; Sexton-Oates, A.; Joo, J.E.; Franchina, M.; Wang, J.J.; Liang, H.; Craig, J.; Saffery, R. DNA methylation landscape of ocular tissue relative to matched peripheral blood. Sci. Rep. 2017, 7, 46330. [CrossRef]

32. Benjamini, Y.; Hochberg, Y. Controlling the False Discovery Rate: A Practical and Powerful Approach to Multiple Controlling the False Discovery Rate: A Practical and Powerful Approach to Multiple Testing. J. R. Stat. Soc. Ser. B Methodol. 1995, 57, 289-300. [CrossRef] 
33. El Khoury, L.Y.; Gorrie-Stone, T.; Smart, M.; Hughes, A.; Bao, Y.; Andrayas, A.; Burrage, J.; Hannon, E.; Kumari, M.; Mill, J.; et al. Systematic underestimation of the epigenetic clock and age acceleration in older subjects. Genome Biol. 2019, 20, 283. [CrossRef]

34. Chen, D.; Chao, D.L.; Rocha, L.; Kolar, M.; Huu, V.A.N.; Krawczyk, M.; Dasyani, M.; Wang, T.; Jafari, M.; Jabari, M.; et al. The lipid elongation enzyme ELOVL2 is a molecular regulator of aging in the retina. Aging Cell 2020, 19, e13100. [CrossRef] [PubMed]

35. Voisin, S.; Harvey, N.R.; Haupt, L.M.; Griffiths, L.; Ashton, K.; Coffey, V.G.; Doering, T.; Thompson, J.-L.; Benedict, C.; Cedernaes, J.; et al. An epigenetic clock for human skeletal muscle. J. Cachex-Sarcopenia Muscle 2020, 11, 887-898. [CrossRef]

36. Castle, J.R.; Lin, N.; Liu, J.; Storniolo, A.M.V.; Shendre, A.; Hou, L.; Horvath, S.; Liu, Y.; Wang, C.; He, C. Estimating breast tissue-specific DNA methylation age using next-generation sequencing data. Clin. Epigenet. 2020, 12, 45. [CrossRef] [PubMed]

37. Ecker, S.; Beck, S. The epigenetic clock: A molecular crystal ball for human aging? Aging 2019, 11, 833-835. [CrossRef]

38. Breton, C.V.; Marsit, C.; Faustman, E.; Nadeau, K.; Goodrich, J.; Dolinoy, D.C.; Herbstman, J.; Holland, N.; LaSalle, J.; Schmidt, R.; et al. Small-Magnitude Effect Sizes in Epigenetic End Points are Important in Children's Environmental Health Studies: The Children's Environmental Health and Disease Prevention Research Center's Epigenetics Working Group. Environ. Health Perspect. 2017, 125, 511-526. [CrossRef]

39. Krämer, A.; Green, J.; Pollard, J.; Tugendreich, S. Causal analysis approaches in Ingenuity Pathway Analysis. Bioinformatics 2014, 30, 523-530. [CrossRef]

40. Geeleher, P.; Hartnett, L.; Egan, L.J.; Golden, A.; Ali, R.A.R.; Seoighe, C. Gene-set analysis is severely biased when applied to genome-wide methylation data. Bioinformatics 2013, 29, 1851-1857. [CrossRef]

41. Foster, K.G.; Acosta-Jaquez, H.A.; Romeo, Y.; Ekim, B.; Soliman, G.A.; Carriere, A.; Roux, P.P.; Ballif, B.A.; Fingar, D.C. Regulation of mTOR Complex 1 (mTORC1) by Raptor Ser863 and Multisite Phosphorylation. J. Biol. Chem. 2010, 285, 80-94. [CrossRef] [PubMed]

42. Zhang, M.; Jiang, N.; Chu, Y.; Postnikova, O.; Varghese, R.; Horvath, A.; Cheema, A.K.; Golestaneh, N. Dysregulated metabolic pathways in age-related macular degeneration. Sci. Rep. 2020, 10, 2464. [CrossRef] [PubMed]

43. Papadopoli, D.; Boulay, K.; Kazak, L.; Pollak, M.; Mallette, F.A.; Topisirovic, I.; Hulea, L. mTOR as a central regulator of lifespan and aging. F1000Research 2019, 8, 998. [CrossRef] [PubMed]

44. Huang, J.; Gu, S.; Chen, M.; Zhang, S.-J.; Jiang, Z.; Chen, X.; Jiang, C.; Liu, G.; Radu, R.A.; Sun, X.; et al. Abnormal mTORC1 signaling leads to retinal pigment epithelium degeneration. Theranostics 2019, 9, 1170-1180. [CrossRef]

45. Krishnamoorthy, J.; Tenkerian, C.; Gupta, J.; Ghaddar, N.; Wang, S.; Darini, C.; Staschke, K.A.; Ghosh, A.; Gandin, V.; Topisirovic, I.; et al. Downregulation of PERK activity and eIF2 $\alpha$ serine 51 phosphorylation by mTOR complex 1 elicits pro-oxidant and pro-death effects in tuberous sclerosis-deficient cells article. Cell Death Dis. 2018, 9, 254. [CrossRef]

46. Scholl, H.P.N.; Issa, P.C.; Walier, M.; Janzer, S.; Pollok-Kopp, B.; Börncke, F.; Fritsche, L.; Chong, V.; Fimmers, R.; Wienker, T.; et al. Systemic Complement Activation in Age-Related Macular Degeneration. PLoS ONE 2008, 3, e2593. [CrossRef]

47. Lu, Y.; Brommer, B.; Tian, X.; Krishnan, A.; Meer, M.; Wang, C.; Vera, D.L.; Zeng, Q.; Yu, D.; Bonkowski, M.S.; et al. Reprogramming to recover youthful epigenetic information and restore vision. Nat. Cell Biol. 2020, 588, 124-129. [CrossRef]

48. Ennis, S.; Jomary, C.; Mullins, R.; Cree, A.; Chen, X.; MacLeod, A.; Jones, S.; Collins, A.; Stone, E.; Lotery, A. Association between the SERPING1 gene and age-related macular degeneration: A two-stage case-control study. Lancet 2008, 372, 1828-1834. [CrossRef]

49. Age-Related Eye Disease Study Research Group The age-related eye disease study system for classifying age-related macular degeneration from stereoscopic color fundus photographs: The age-related eye disease study report number 6. Am. J. Ophthalmol. 2001, 132, 668-681. [CrossRef]

50. Aryee, M.J.; Jaffe, A.E.; Corrada-Bravo, H.; Ladd-Acosta, C.; Feinberg, A.P.; Hansen, K.D.; Irizarry, R.A. Minfi: A flexible and comprehensive Bioconductor package for the analysis of Infinium DNA methylation microarrays. Bioinformatics 2014, 30 1363-1369. [CrossRef]

51. Koestler, D.C.; Jones, M.J.; Usset, J.; Christensen, B.C.; Butler, R.A.; Kobor, M.S.; Wiencke, J.K.; Kelsey, K.T. Improving cell mixture deconvolution by identifying optimal DNA methylation libraries (IDOL). BMC Bioinform. 2016, 17, 120. [CrossRef]

52. Chen, Y.-A.; Lemire, M.; Choufani, S.; Butcher, D.T.; Grafodatskaya, D.; Zanke, B.W.; Gallinger, S.; Hudson, T.J.; Weksberg, R. Discovery of cross-reactive probes and polymorphic CpGs in the Illumina Infinium HumanMethylation450 microarray. Epigenetics 2013, 8, 203-209. [CrossRef]

53. Pidsley, R.; Zotenko, E.; Peters, T.J.; Lawrence, M.G.; Risbridger, G.P.; Molloy, P.; Van Djik, S.; Muhlhausler, B.; Stirzaker, C.; Clark, S.J. Critical evaluation of the Illumina MethylationEPIC BeadChip microarray for whole-genome DNA methylation profiling. Genome Biol. 2016, 17, 208. [CrossRef]

54. Maksimovic, J.; Gordon, L.; Oshlack, A. SWAN: Subset-quantile within array normalization for illumina infinium HumanMethylation450 BeadChips. Genome Biol. 2012, 13, R44. [CrossRef] [PubMed]

55. Ritchie, M.E.; Phipson, B.; Wu, D.; Hu, Y.; Law, C.W.; Shi, W.; Smyth, G.K. limma powers differential expression analyses for RNA-sequencing and microarray studies. Nucleic Acids Res. 2015, 43, e47. [CrossRef] [PubMed]

56. Peters, T.J.; Buckley, M.J.; Statham, A.L.; Pidsley, R.; Samaras, K.; Lord, R.V.; Clark, S.J.; Molloy, P.L. De novo identification of differentially methylated regions in the human genome. Epigenet. Chromatin 2015, 8, 6. [CrossRef] [PubMed] 